DOI: $\underline{10.23857 / \text { pc.v4i9.1102 }}$

\title{
La viabilidad ambiental: Una mirada desde los proyectos socio integradores
}

The environmental viability: A look from the integrating partner projects

\section{A viabilidade ambiental: Uma visão dos projetos parceiros integradores}

\author{
Lucrecia Corzo de Rodríguez ${ }^{\mathrm{I}}$ \\ lucrecorzo@hotmail.com \\ https://orcid.org/0000-0001-8125-5053 \\ Jeanmary Chirinos ${ }^{\text {II }}$ \\ lucrecorzo@hotmail.com
}

\section{Correspondencia: lucrecorzo@hotmail.com}

Ciencias de la educación

Artículo de investigación

*Recibido: 15 de Julio de 2019*Aceptado: 13 de Agosto de 2019* Publicado: 05 de Septiembre 2019

I. Docente de la Universidad Politécnica Territorial de Falcón Alonso Gamero, Departamento de Proyectos, Santa Ana de Coro, Venezuela.

II. Docente de la Universidad Politécnica Territorial de Falcón Alonso Gamero, Departamento de Proyectos, Santa Ana de Coro, Venezuela. 


\title{
Resumen
}

Los Proyectos Socio Integradores (PSI) que se vienen realizando en la Universidad Politécnica Territorial de Falcón Alonso Gamero (UPTAG), se caracterizan porque se conciben como el direccionamiento de la manifestación y voluntad colectiva de las comunidades organizadas, surgen a partir de los problemas o potencialidades territoriales diagnosticadas, es por ello que nace el propósito de esta investigación: Develar el significado que le otorgan los estudiantes de química a la construcción de la VA en los PSI realizados en los últimos cinco años, la investigación se enmarca en el paradigma interpretativo, con un enfoque cualitativo, empleando el método hermenéutico. Los hallazgos develan un patrón de debilidades y falta de claridad conceptual de la VA. Se requiere cambios en la guía orientadora, así como formar a los docentes en la aplicación del eje transversal para garantizar egresados con conciencia humanística, ética y responsabilidad ambiental.

Palabras Claves: Educación de adultos; enseñanza superior; estudiante universitario; plan de estudios universitario.

\begin{abstract}
The Socio Integradores Projects (PSI) that are being carried out at the Falcon Alonso Gamero Territorial Polytechnic University (UPTAG), are characterized because they are conceived as the direction of the manifestation and collective will of the organized communities, arising from the problems or Territorial potentialities diagnosed, that is why the purpose of this research is born: Reveal the meaning that chemistry students give to the construction of the VA in the PSI carried out in the last five years, the research is framed in the interpretative paradigm, with a qualitative approach, using the hermeneutical method. The findings reveal a pattern of weaknesses and lack of conceptual clarity of the VA. Changes in the guidance guide are required, as well as training teachers in the application of the transversal axis to guarantee graduates with humanistic awareness, ethics and environmental responsibility.
\end{abstract}

Keywords: Adult education; Higher education; University students; University curriculum.

\section{Resumo}

Os Projetos Socio Integradores (PSI) que estão sendo realizados na Universidade Politécnica Territorial do Falcon Alonso Gamero (UPTAG), caracterizam-se por serem concebidos como 
direção da manifestação e vontade coletiva das comunidades organizadas, decorrentes de problemas ou problemas territoriais. potencialidades diagnosticadas, por isso nasce o objetivo desta pesquisa: Revelar o significado que os estudantes de química atribuem à construção do VA no PSI realizado nos últimos cinco anos, a pesquisa está enquadrada no paradigma interpretativo, com uma abordagem qualitativa. abordagem, utilizando o método hermenêutico. Os resultados revelam um padrão de fraquezas e falta de clareza conceitual do VA. São necessárias mudanças no guia de orientação e treinamento de professores na aplicação do eixo transversal para garantir aos graduados consciência humanística, ética e responsabilidade ambiental.

Palavras-chave: Educação de adultos; ensino superior; estudantes universitários; currículo universitário

\section{Introducción}

Las razones que dan origen a un proyecto pueden ser diversas, una necesidad, un problema, la producción de bienes y servicios, el aprovechamiento de materias primas potenciales, emprendimientos, en fin es una iniciativa de negocio e inversión, sin embargo a lo largo de la historia hemos sido testigo de que la mayoría de los proyectos que llegan a cristalizarse, prevalece la relación capitalista y rentista sobre la visión proteccionista del ambiente, puesto que los elementos ambientales no son tomado como factor de progreso, tal como lo sustenta Varsavsky (2007) la idea de desarrollo, es la extractiva y sobre explotadora de los recursos naturales al servicio de una sociedad cada vez más consumista de necesidades creadas.

En este sentido, analizar y construir la viabilidad ambiental de un proyecto en su fase de planificación, es una garantía de protección del ambiente y de desarrollo sostenible, son actividades complementarias, pues no puede existir progreso si éste no va de la mano con la conservación ambiental. De acuerdo a lo anterior, los PSI, que se vienen realizando en la UPTAG, como parte el desarrollo curricular enmarcados en el paradigma socio crítico, se caracterizan porque se conciben como el direccionamiento de la manifestación y voluntad colectiva de las comunidades organizadas, puesto que surgen a partir de los problemas o potencialidades territoriales diagnosticadas pero también se caracterizan porque significan un compromiso entre los que participan (docentes, estudiantes, comunidades, instituciones) en un proceso de cambio de la sociedad en la cual vivimos. 
Estos PSI además de ser un referente de la trasformación social también aglutinan acciones que intervienen en los elementos ambientales particulares de cada territorio, por lo que los responsables del PSI, en la etapa de planificación, deben caracterizar el establecimiento de las relaciones eco-ambientales y el proyecto, estableciendo lo que se conoce como la viabilidad ambiental del mismo, analizando las múltiples dimensiones que el ambiente manifiesta y como el proyecto puede asimilarse al medio con el menor efecto negativo, previendo si existe esa compatibilidad entre el proyecto-ambiente, los participantes estarán en capacidad de decidir que el proyecto es viable ambientalmente.

Lo anterior es sumamente importante para cumplir con uno de los principios y valores en las experiencias de formación; "la educación universitaria sea para la defensa ambiental y de su equilibrio ecológico en todos los ámbitos territoriales en función de un desarrollo sustentable" lo que es fundamental para contribuir a la formación integral de individuos, que no repitan conductas depredadoras, irresponsables, que rompan el equilibrio ecológico de sus comunidades. Sobre las ideas planteadas anteriormente, surge el tema para esta investigación libre, cuyo propósito se direcciona hacia la dilucidación de cómo construyeron los participantes (estudiantes) en calidad de investigadores del PNF de Química y de Procesos Químicos la Viabilidad Ambiental en los PSI.

\section{Aproximación al objeto de estudio}

En el ejercicio de la práctica docente, nos enfrentamos diariamente a una multitud de responsabilidades, entre ellas la de ser asesor técnico o jurado evaluador de producciones escritas concebidos como PSI, siendo éste un compromiso que se convierten en una oportunidad para aproximarnos a una nueva manera de investigar, a través de la interacción con las comunidades, acercándonos a leer la realidad social, humanizando la investigación, donde se generan nuevas experiencias contextualizadas en pro de la construcción de conocimiento, es así pues, desde esa ventana, ejerciendo la figura de jurado evaluador, mientras revisaba el PSI, cuando nace el interés de investigar de la sección conocida como viabilidad del proyecto, específicamente la referida a la VA.

En esa revisión, la VA presentaba deficiencias tales como; la exposición escrita la hacía en un párrafo de cuatro líneas, la descripción de los alcances eran incompletos, la información sobre qué actividades del proyectos podrían degradar el entorno era escueta y los elementos abióticos y 
bióticos presentes en cualquier comunidad no eran mencionados en su totalidad, esto me hizo preguntarme si ¿las carencias observadas en la construcción de la viabilidad ambiental será recurrentes?, ¿Qué elementos teóricos y prácticos se necesitan para construir la VA de cualquier proyecto?, ¿Cómo caracterizan la relación proyecto-ambiente en la comunidad donde intervienen los estudiantes?.

Para aproximarnos a las posibles respuestas, inicialmente sostuvimos una conversación informal con una representante del comité departamental de proyecto, a quien le preguntamos si existían algunas directrices desde este comité para construir la VA, a lo que esta profesora nos respondió "la construcción de la VA en los PSI, se encuentra cercada por un alcance específico para cada trayecto, esta información está disponible en una guía orientadora, la cual debe ser suministrada a los estudiantes, por el profesor de la unidad curricular de proyecto," también no dijo que estos alcances fueron construidos en un trabajo en equipo, éstos fueron discutidos y consensuados por el conjunto de profesores del Departamento Académico de Química (DAQ) que conforman el comité mencionado anteriormente" cabe señalar que los alcances se muestran a continuación en el cuadro número uno (1). 
Alcance de la VA por trayecto para cada PNF

PNF PROCESOS

Cuadro 1

QUÍMICOS

TRAYECTO ALCANCE DE LA VA

\begin{tabular}{ll}
\hline I & $\begin{array}{l}\text { Identificación preliminar de potenciales contaminantes- caracterización } \\
\text { físico-natural }\end{array}$ \\
II & Identificación de efluentes contaminantes y sustancias toxicas \\
III & Prevé las medidas de control y saneamiento ambiental \\
IV & $\begin{array}{l}\text { Presenta alternativas de procesos para los posibles tratamientos de efluentes } \\
\text { y sólidos contaminantes }\end{array}$
\end{tabular}

\section{PNF QUÍMICA}

TRAYECTO

I

II

III

IV

\section{ALCANCE DE LA VA}

Identificación de los posibles contaminantes en la comunidad y/o de la materia prima

Especificar los contaminantes de la materia prima y los posibles a generar en el producto terminado (subproductos-derivados de la síntesis)

Tomar en cuenta en el desarrollo del proyecto las medidas de control y saneamiento ambiental

Presenta alternativas para el tratamiento de los desechos generados en la ejecución del nuevo producto y/o método desarrollado

Fuente: Comité de Proyecto del Departamento Académico de Química, (PNF-Procesos Químicos, 2017) (PNF-Química, 2017)

Por otro lado, para darle cuerpo a la idea de investigación, emprendimos una búsqueda al pasado reciente del DAQ, donde consideramos la lectura de los PSI realizados desde este departamento, en el periodo de marzo de 2012 hasta marzo del año 2017, conciliando el conocimiento como el resultado de una dialéctica entre el sujeto conocedor y el objeto conocido, indagando si estos proyectos ya defendidos y evaluados por docentes y comunidades, representaban una evidencia de la deficiencias advertidas en la revisión que motivó la investigación, buscando si acaso se repetían patrones o por el contrario era solo un caso atípico, es allí en donde radica la importancia que tiene la investigación pues revisaremos el significado que ha tenido la VA para los estudiantes, en los últimos cinco años como parte de una realidad socioeducativa para ello nos trazamos los siguientes propósitos de investigación. 


\section{Propósitos de la investigación}

\section{Propósito General:}

Comprender el significado asumido por los participantes del PNF de Química y de Procesos Químicos en la construcción de la Viabilidad Ambiental en los PSI.

\section{Propósitos Específicos:}

Develar el significado que le otorgan los participantes en la construcción de la VA en los PSI.

Describir la existencia de nexos entre los PSI realizados en el PNF de Química y de Procesos Químicos en el periodo 2012-2017

Interpretar las tendencias asumidas por los participantes en la construcción de la VA en los PSI

\section{Referentes teóricos}

Para entrar en contexto con el tema de la investigación, comenzaremos con ilustrar el significado del vocablo viabilidad, el cual hace referencia en el diccionario de la Real Academia Española; a lo viable, concepto que proviene de vie, vida, indica que puede vivir, desarrollarse, refiere a “...un asunto que, por sus circunstancias, tiene probabilidades de poderse llevar a cabo", por otra parte, en relación al logro de cualquier iniciativa de negocio o proyecto, en palabras de Ortegón, (2005) considera que Viabilidad indica lo que puede concretarse, aquello que puede salvar los escollos esperables o no, en el recorrido necesario para materializarse.

En este mismo sentido, Ocampo (2003) señala que en el caso de Proyectos de Inversión puede entenderse por viabilidad "la capacidad de un proyecto de asimilarse al medio intervenido y transformarlo, en forma sostenible". Siguiendo la misma tónica, ahora nos referiremos al término ambiente, el cual lo interpretamos de una forma amplia y globalizadora como un conjunto de interacciones entre los factores bióticos (que tienen vida ej. especie humana, animal y vegetal), abióticos (sin vida ej. luz solar, aire, agua), geofísicos, tecnológicos, económicos, sociales, políticos, culturales y estéticos en permanente transformación; de allí pues que como sociedades nos desarrollamos dentro de ese entorno de interrelaciones y a lo largo de la historia hemos modificado, como consecuencia el medio ha respondido a esos cambios limitando y condicionando nuestro comportamiento para el alcance de una determinada calidad de vida.

De lo anterior, podemos derivar a la frase Viabilidad Ambiental referida a un proyecto, como la expresión que relaciona la capacidad del ambiente de recuperarse ya sea por medios naturales o 
artificiales y de los promotores de los proyectos de hacer un manejo adecuado de las alteraciones positivas o negativas; de tal forma, que se pueda garantizar un nuevo equilibrio proyectoambiente, ante la intervención de cualquier actividad humana que implique producción o prestación de bienes y servicios, suministro de materias primas y/o desarrollo de infraestructura. Lo que nos hace entender que es necesario que entre los PSI y el medio que se propone intervenir se establezcan relaciones eco-ambientales amigables, es decir que sean compatibles, consistentes, adaptables y por supuesto sustentables.

En otro orden de ideas, en la UPTAG, de acuerdo a las nuevas mallas curriculares de ambos PNF, los estudiantes reciben información del aspecto ambiental a lo largo del proceso académico, temas como legislación ambiental, descripción de los elementos que conforman el ambiente, las actividades de la ciencia y tecnología susceptible de degradar el ambiente entre otros, son abordados en la unidad curricular ambiente y desarrollo sustentable, en la cual además de intercambiar estos saberes con los estudiantes, éstos contenidos deben articularse al eje transversal proyecto.

En este sentido, es importante recordar que el ambiente es ideado como un eje transversal, en la Ley orgánica de educación (2009), tal como lo reza el numeral 5 del artículo 15 de la mencionada ley: uno de los fines de la educación es "Impulsar la formación de una conciencia ecológica para preservar la biodiversidad y la socio diversidad, las condiciones ambientales y el aprovechamiento racional de los recursos naturales". De allí pues, entendemos que los docentes de todas las unidades curriculares, deben tributar los contenidos de cada disciplina a la temática ambiental, relacionando de manera intencional y contextualizada a nivel local, regional y nacional todas las problemáticas y potencialidades de nuestro ambiente, de tal manera que se garantice que el tópico atraviese todo el currículo y se cumpla con el perfil de egreso de los PNF “...formar individuos con actitud crítica, reflexiva, con disposiciones responsable, éticovalorativas hacia el ambiente" en esta línea se sustenta teórica y legalmente la investigación en curso.

\section{Ruta metodológica}

Atendiendo a la naturaleza objeto de estudio, el significado que le otorgan los estudiantes a la construcción de la VA en los PSI, la cual es variada de múltiples expresiones, como diversas son las comunidades con sus realidades, problemáticas, sus necesidades y las probables soluciones 
que se ofrecen a través de los PSI. De allí pues, que la mejor manera de acercarse a lo planteado anteriormente es a través del paradigma interpretativo, el cual según Tójar (2006) "valoriza la visión que sobre ellos mismos tienen los sujetos, los significados de los comportamientos implícitos y explícitos" (p.62). En este sentido nos apoyamos en el enfoque cualitativo para comprender la realidad de estudio, tal como nos indica Silva (2007), que es la "Construcción de un conocimiento de la realidad social y cultural desde el punto de vista de quienes la producen, la reproducen y la viven" (p.23) en este caso los estudiantes a través de los textos plasmados en los PSI.

En cuanto al método aplicado para estudiar la realidad en la presente investigación se inicia con un recorrido histórico, al respecto, Gadamer (1996) indica que "una hermenéutica adecuada debe mostrar en la comprensión misma la realidad de la historia" (p.370) considerando que la realidad abordada en cuestión es influenciada por un amplio recorrido histórico de los PSI elaborados en los pasados cinco años, lo que interviene en el significado conferido a la construcción de la VA y las tendencias asumidas a lo largo de ese periodo. Para alcanzar los propósitos de la investigación aplicamos la técnica de Análisis de Contenido, que de acuerdo a Díaz y Navarro (1998), es una técnica de investigación que utiliza un conjunto de procedimientos tanto cualitativos como cuantitativos para hacer inferencias reproducibles y válidas a partir de un texto (de los datos al contexto de los mismos) con la finalidad de describir las tendencias y cambios en el contenido de los documentos.

En consecuencia los textos analizados son las producciones intelectuales escritas, consideradas material original e inédito de los autores (los estudiantes), pensadas como fuentes primarias, convirtiéndose de este modo en la fuente directa de insumos de la investigación, y de un único origen común los PNF de Q y de PQ. Para efecto de esta investigación, el universo de estudio o universo de datos, consideré todos los PSI almacenados en la biblioteca electrónica del laboratorio de computación del DAQ correspondientes a ambos PNF, representando éstos, un total de cincuenta y ocho (58) proyectos guardados en discos compactos (CD). Las producciones escritas las fuimos ordenando cronológicamente y separándolas por PNF, quedando discriminada de la siguiente manera: cuarenta y cinco (45) proyectos de procesos químicos (PQ) y trece (13) proyectos de química $(\mathrm{Q})$, seguidamente hicimos otra agrupación de acuerdo al objeto de estudio de cada PSI, codificándolos de la siguiente manera: Diseño de Planta (DP); Construcción de Equipo (CE), Trasformación de Materia Prima (TMP), Mejoras Operacionales de Procesos 
(MOP) y Otros (O). Con el uso de esta codificación pudimos esclarecer que en DP se ubican diecisiete (17) proyectos, en CE se sitúan doce (12), para TMP y MOP se encuentran siete (7) y seis (6) respectivamente para el PNF de PQ.

Por su parte en el PNF de Q, once proyectos se ubican en la TMP y dos en Otros. Con relación al procesamiento de la información y credibilidad de la investigación, se realizó una triangulación, para lo cual, primero, a través de las técnicas de análisis de contenido, surgieron las categorías identificadas en el recorrido histórico de los PSI, se ubicaron las distintas categorías en la metodología aplicada en la elaboración de la VA por Jorge Arboleda (2008) y en la guía orientadora del DAQ, donde se detallan las directrices que deben alcanzar los estudiantes en la construcción de la VA por trayecto, los cuales se sometieron a una triangulación para contrastar los datos surgidos de la técnica de análisis de contenido y la información ya referenciada.

\section{Hallazgos de la investigación}

En esta sección del extenso procedemos a mostrar los resultados plasmados en cuadros, que se elaboran posterior a cumplir las fases de lectura de los sesenta y cuatro PSI, ubicación de la unidad de análisis en el texto (VA), localizar dentro texto lo sobresaliente de las unidades de análisis delimitándose como las categorías y subcategorías que emergen del texto, a continuación se detalla la información para el PNF de PQ. Para el año 2012, los estudiantes construyen la VA basada principalmente a los beneficios que la implementación del proyecto traerá a la comunidad $y$ al ambiente en general,

Igualmente indican que el empleo de técnicas o tecnologías sustentables hacen viable el PSI, otros grupos señalan que la no generación de desechos peligrosos hacen viable el proyecto, de igual modo recurren a la factibilidad del proyecto como parte de la VA, un grupo construye la unidad de análisis aplicando una metodología adecuada para la VA, señalando que el proyecto genera un impacto ambiental bajo, analizaron tanto en la fase de construcción como en la fase de operación, recomendando algunas medidas de mitigación, lo que corresponde con el alcance exigido por el DAQ.

En el año 2013, un solo grupo aplica una de las metodologías disponible para estos casos, como es la matriz de doble entrada de valoración cualitativa de impactos, permitiéndoles caracterizar los efectos que produciría el proyecto sobre el medio y recomendar medidas de mitigación de los mismos. Por otra parte el resto de los grupos relacionan la VA con los beneficios que dejará el 
proyecto sobre el ambiente puesto que no se producen desechos que perjudiquen al mismo, así también un grupo asocia un proceso cuyas actividades son artesanales (la elaboración del licor de Agave Cocui un proceso que genera altas tasa de vinaza como subproducto potencialmente contaminante el cual es ácido y es descargado directamente al suelo y a cuerpos de agua) con el hecho de que no causan contaminación por lo que resulta viable su PSI.

Por su parte, la gran mayoría de los PSI realizados en el año 2014 se caracterizaron por asociar la VA con la resolución de una problemática de índole ambiental, relacionado la situación actual que ellos encuentran cuando efectúan el diagnostico con una situación ideal que esperan cambiar con la implementación del proyecto. Otros grupos relacionan la VA con la no generación de impactos al ambiente porque no generan desechos peligrosos. Un grupo identifica algunos impactos a priori sin mencionar aplicación de metodología y otro señala que su PSI es viable porque cumple con un artículo de la ley orgánica del ambiente.

En el año 2015, pudimos observar que dos grupos aplican la metodología de matriz de Leopold y de valoración de Delladova, abordando el proyecto en pleno (construcción y operación) y la recomendación de medidas de mitigación y control. Otros dos grupos señalan la VA con la producción de beneficios y a la resolución de una problemática de índole ambiental, siendo este enfoque necesario fomentar en las universidades politécnicas tal como plantea Guanipa Gotopo (2018).

Por su parte, en la continuación del cuadro dos, podemos observar que para el año 2016, que un grupo aplica una metodología para conocer si el proyecto es viable o no, en este caso usaron la Lista de chequeo, identificaron los efectos que produciría el proyecto (Fase de construcción y operación) Identifican los efluentes que se generan en su planta, y proponen medidas de saneamiento ambiental al proponer salidas útiles y aprovechables para estos desechos.

Uno de los grupos expone que su PSI es viable porque cumplen normas de Seguridad, Higiene y Ambiente para evitar accidentes laborables, otro grupo relacionan factibilidad y beneficios del proceso con la VA del proyecto. Por otro lado, observamos que otro grupo identifica a priori los efluentes de su equipo y propone medidas para mitigar los efectos de estos efluentes con aprovechamiento útil de los mismos, cumpliendo a medias con los alcances propuestos por el DAQ, en el resto de los grupos se observa que expresan en sus producciones intelectuales una reciprocidad entre la VA, la no generación de desechos peligrosos y la resolución de un problema con la aplicación de una tecnología amigable con el ambiente.

\section{8}

Pol. Con. (Edición núm. 37) Vol. 4, No 9, Septiembre 2019, pp. 108-132, ISSN: 2550 - 682X 
Categorías emergentes del corpus texto de la unidad de análisis Viabilidad Ambiental (VA) en los PSI del PNF de Procesos Químicos

$\begin{array}{lccc}\text { Código } & \text { Unidad de Análisis } & \text { Sub-Categoría } & \begin{array}{r}\text { Categoría } \\ \text { de PSI }\end{array} \\ & \text { Emergente }\end{array}$

\begin{tabular}{|c|c|c|}
\hline $\begin{array}{l}\text { 1-PQ- } \\
2012- \\
\text { CE }\end{array}$ & $\begin{array}{l}\text { "el uso de la biomasa para producir energía } \\
\text { traerá muchos beneficios ambientales a la } \\
\text { comunidad"(pp-43) }\end{array}$ & Beneficios al ambiente \\
\hline $\begin{array}{l}2-P Q- \\
2012- \\
\text { CE }\end{array}$ & $\begin{array}{l}\text { "el tratamiento de los desechos sólidos es viable } \\
\text { porque contribuye al mejoramiento ambiental" } \\
\text { (pp-46) }\end{array}$ & $\begin{array}{l}\text { Mejoramiento } \\
\text { ambiental }\end{array}$ \\
\hline $\begin{array}{l}\text { 3-PQ- } \\
\text { 2012- } \\
\text { TMP }\end{array}$ & $\begin{array}{l}\text { "el reciclaje de desechos sólidos para } \\
\text { transformarlo en un producto útil, se traduce en } \\
\text { un importante beneficio que concientiza a la } \\
\text { comunidad por lo que el proyecto es } \\
\underline{\text { factible"(pp-41) }}\end{array}$ & $\begin{array}{l}\text { Beneficio a la } \\
\text { comunidad } \\
\text { El proyecto es factible }\end{array}$ \\
\hline $\begin{array}{l}\text { 4-PQ- } \\
\text { 2012- } \\
\text { CE }\end{array}$ & $\begin{array}{l}\text { "el estudio de Factibilidad y viabilidad del } \\
\text { diseño...dispone de los servicios necesarios } \\
\text { para su construcción, y en la operación no se } \\
\text { visualizan desechos sólidos peligrosos en este } \\
\text { proyecto"(pp-52) }\end{array}$ & $\begin{array}{l}\text { Factibilidad } \\
\text { Fase de construcción } \\
\text { Fase de operación } \\
\text { No se generan desechos } \\
\text { peligrosos }\end{array}$ \\
\hline
\end{tabular}

5-PQ- "se seleccionó la tecnología más

2012-ST adecuada....siendo uno de los criterios el impacto ambiental.. seleccionando el humedal como la más viable “ (pp-51)

Tecnología amigable con el ambiente

Beneficios

Beneficios

Factibilidad

Factibilidad

Identificación de materia primas

No se generan desechos

Empleo de
técnicas $\quad$ o
tecnologías
sustentables

No se generan desecho peligrosos

6-PQ- "el estudio de viabilidad nos indica la 2012- generación de un impacto ambiental...que DP contempla el desmalezamiento... Las aguas de descarga...pueden descargarse al mar o recomendar su uso para riego previa evaluación" (p"-54)

Se genera impacto ambiental

Aplicación de una

Fase de construcción metodología para construir

Fase de operación la VA

Medidas de mitigación

Beneficios al ambiente

Beneficios

7-PQ- “es viable ambientalmente porque traerá

MOP energético"(pp-42)

8-PQ- "el proyecto es viable para el ambiente porque

Beneficios al ambiente

Beneficios

2013- $\quad$ se deja de usar aditivos químicos y estos se

Sustitución de reactivos 
TMP sustituyen por aditivos naturales"(pp-44)

9- PQ- "la construcción de la planta no acarrea ningún

2013- impacto negativo al ambiente no se va afectar al

DP ambiente"(pp-39)

10-PQ- "es viable porque en este equipo que no hay

2013- generación de desechos que perjudiquen al

CE ambiente" $(\mathrm{pp}-40)$

11-PQ- "Es viable porque el proyecto se apoya en una

2013- actividad artesanal que contribuye al desarrollo

CE endógeno sostenible y sustentable en el tiempo"(pp-45)

12-PQ- $\quad$ No aparece la unidad de análisis VA en el PSI 2013-ST

13-PQ- "se realizó una matriz de doble entrada, 2013- obteniéndose que el proyecto generará impacto

DP moderados a tener en cuenta en el ambiente natural, en la calidad del aire y del suelo, por lo que se proponen medidas de mitigación de estos efectos"(pp-55)

14-PQ- "el proyecto es viable porque hay sustitución de 2013-

MOP

15-PQ2013-

CE

16-PQ- $\quad$ No aparece la unidad de análisis VA en el PSI 2013-

DP

17-PQ- "es viable porque el equipo no genera residuos

2014- solido o líquidos indeseables"(pp-43)

$\mathrm{CE}$

18-PQ- "la planta procesadora de leche no genera

2014- impacto ambiental porque se basa en el

DP aprovechamiento sostenible de recursos naturales con interés agroalimentario"(pp46)

19-PQ- "es viable porque el proyecto cumple con el 2014- artículo 36 de la ley orgánica del ambiente: $\mathrm{CE}$ aquellas personas que formulen un proyecto que implique el uso de recursos naturales deben tóxicos

Fase de construcción Impacto

No genera impactos negativos

No hay generación de desechos

No se generan desechos

\section{Actividad artesanal Proceso} Artesanal

Matriz de doble entrada

Impacto ambiental

moderado

Medidas de mitigación

Beneficios al ambiente

Sustitución de reactivos tóxicos

Es viable

No se generan

No produce

emanaciones

Es viable

No se generan

No genera residuos

sólidos o líquidos

No se genera impacto

ambiental

Aprovechamiento

Es viable porque

cumple con la LOA
Beneficios

desechos

desechos

Impactos

Asociada a

Aplicación de metodología para construir la VA

desechos

Leyes 
generar procesos permanentes de educación ambiental"'(pp-51)

20-PQ- " "la tala de árboles no será de grandes 2014- extensiones, no se destruyen muchos hábitats DP por eso es viable"(pp-42)

Tala de arboles

Destrucción de hábitats

Es viable

Poca afectación ecológica
Identificación de impactos

No se generan desechos

21-PQ- "con el proyecto se prevé poca afectación de las condiciones ecológicas de la zona, no se usaran reactivos químicos que generen preocupación en el proceso" $(p p-40)$

\section{Cuadro 2}

$\begin{array}{llcc}\begin{array}{l}\text { Código } \\ \text { de PSI }\end{array} & \text { Categoría } & \text { Categoría } \\ & & \text { Emergente } & \end{array}$

\begin{tabular}{|c|c|c|c|}
\hline $\begin{array}{l}22-P Q- \\
2014-\end{array}$ & $\begin{array}{l}\text { "el recubrimiento de las tuberías disminuirá la } \\
\text { contaminación térmica al ambiente"(pp-41) }\end{array}$ & $\begin{array}{l}\text { Disminución se la } \\
\text { contaminación }\end{array}$ & $\begin{array}{l}\text { Resolución de un } \\
\text { problema }\end{array}$ \\
\hline $\begin{array}{l}23-\mathrm{PQ}- \\
2014- \\
\text { TMP }\end{array}$ & $\begin{array}{l}\text { "es viable porque se evita la degradación del } \\
\text { ambiente por el desaprovechamiento de las } \\
\text { naranjas" (pp-49) }\end{array}$ & $\begin{array}{l}\text { Es viable } \\
\text { Evita la Degradación del } \\
\text { ambiente }\end{array}$ & $\begin{array}{l}\text { Resolución de un } \\
\text { problema }\end{array}$ \\
\hline $\begin{array}{l}\text { 24-PQ- } \\
2014- \\
\text { TMP }\end{array}$ & $\begin{array}{l}\text { "es viable ambientalmente porque se reduce } \\
\text { considerablemente los plástico desechados en la } \\
\text { comunidad"(pp-44) }\end{array}$ & $\begin{array}{l}\text { Es viable } \\
\text { Reducción de desechos }\end{array}$ & $\begin{array}{l}\text { Resolución de un } \\
\text { problema }\end{array}$ \\
\hline $\begin{array}{l}\text { 25-PQ- } \\
\text { 2014- } \\
\text { TMP }\end{array}$ & No aparece la unidad de análisis VA en el PSI & & \\
\hline $\begin{array}{l}\text { 26-PQ- } \\
2014- \\
\text { TMP }\end{array}$ & $\begin{array}{l}\text { "no se generan desechos en el proceso....se } \\
\text { impulsa el uso de envases reciclados creando } \\
\text { conciencia de reciclaje"(pp-47) }\end{array}$ & $\begin{array}{l}\text { No se generan desechos } \\
\text { Conciencia del reciclaje }\end{array}$ & $\begin{array}{l}\text { No se generan } \\
\text { desecho } \\
\text { peligrosos }\end{array}$ \\
\hline $\begin{array}{l}27-P Q- \\
2014- \\
\text { DP }\end{array}$ & No aparece la unidad de análisis VA en el PSI & & \\
\hline $\begin{array}{l}\text { 28-PQ- } \\
\text { 2014-ST }\end{array}$ & $\begin{array}{l}\text { "con el humedal se soluciona el problema de } \\
\text { malos olores, proliferación de mosquitos" (pp- } \\
\text { 46) }\end{array}$ & $\begin{array}{l}\text { Solución de } \\
\text { problemáticas }\end{array}$ & $\begin{array}{l}\text { Resolución de un } \\
\text { problema }\end{array}$ \\
\hline $\begin{array}{l}29-P Q- \\
2014-\end{array}$ & $\begin{array}{l}\text { "se espera que con el proyecto la comunidad se } \\
\text { eduque en el reciclaje del vidrio y este no se }\end{array}$ & Reducción de vidrios & Resolución de un \\
\hline
\end{tabular}


MOP

desecha como se observa actualmente" (pp-48)

30-PQ- "es viable ambientalmente porque se reduce

2014-

TMP

31-PQ-

2014-

MOP

32-PQ- $\quad$ No aparece la unidad de análisis VA en el PSI

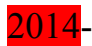

DP

33-PQ- "La tecnología seleccionada es viable porque el

2014-

CE

34-PQ- "se realizó una matriz de valoración cualitativa 2015-

DP considerablemente los plástico desechados en la comunidad"(pp-44)

"a través de este proyecto se reduce la problemática de contaminación generada por el proceso de curtimbre"(pp-54) producto obtenido es menos toxico y tiene características de biodegradabilidad"

de impactos, evidenciándose un listado de efectos razonables producto de la construcción de la planta y la red de distribución"(pp-54)

Reducción de la problemática de contaminación

Producto obtenido es menos toxico y biodegradable

Matriz de valoración cualitativa de impactos

Lista de efectos

Fase de construcción

Fase de operación

35-PQ- "se construyó una matriz de Leopold, en la cual 2015- se cruza la magnitud, la extensión del área con DP el valor del impacto para la construcción de la planta"(pp-57)

36-PQ- "la disminución de la leña usada como 2015- combustible, es un beneficio notorio que MOP producirá el proyecto" (pp-47)

37-PQ- "el aprovechamiento de estiércol para producir 2015-

DP

38-PQ- "la planta no es muy amplia por lo que no será 2016- mucho el impacto......con esta planta se mejora DP la calidad de vida de los habitantes...los efectos ocasionados se mitigarán con prácticas ambientales que compaginen con el ambiente"(pp-54)

39-PQ- "se toma en cuenta todo los efectos que tendrá 2016- la construcción de la planta, en una lista de DP chequeo,...el movimiento de tierra, el cambio del relieve, excavación de zanjas, construcción de trillas, maquinarias pesadas, generación de

Matriz de Leopold

Listado de efectos

Fase de construcción

Beneficio notorio del

proyecto

Solución viable

Proceso biodegradable

Poco impacto

Mejora la calidad de vida

Mitigación de efectos

Lista de chequeo

Efectos del proyecto

Fase de construcción problema

Resolución de un problema

Resolución de un problema

Resolución de un problema

Aplicación de una metodología para construir la VA

Aplicación de una metodología para construir la VA

Beneficios

Resolución de un problema

Beneficios

Aplicación de una metodología para construir la VA 
ruidos, colocación de tuberías....los desechos generados durante la operación de la planta tienen salida positiva de la planta porque pueden comercializarse" (pp-45)

40-PQ- "con la tecnología seleccionada se reduce los 2016- residuos de poda que la comunidad arroja a los CE terrenos baldíos....el equipo genera desechos de alquitrán que pueden ser aprovechados como impermeabilizantes, las cenizas como fertilizantes...”(pp-56)

41-PQ- "la tecnología seleccionada es viable porque no 2016- interviene significativamente el ambiente, no se DP genera impactos negativos en la comunidad"(pp-46)

42-PQ- "El secado de bandejas es una propuesta 2016 didáctica que no causa contaminantes al medio $-\mathrm{CE}$

43-PQ- "se cumplen con todas las normas de cuidado y 2016- protección del medio ambiente con el objetivo CE de evitar accidentes laborales" (pp-49)

44-PQ- "el biodigestor es una tecnología amigable con 2016- el medio ambiente, no genera efectos negativos CE al medio ambiente"(pp-38)

45-PQ- "el procesamiento de la leche traerá más 2016- beneficios a la comunidad y al ambiente que DP consecuencias, por lo que es factible el proyecto"
Fase de operación

Generación de desechos

Salida positiva a los

desechos

Resolver una

Resolución de problemática Generación problemas de desechos

Que pueden ser aprovechables

Medidas de mitigación

No interviene al ambiente

No genera impacto negativos

No causa contaminantes al ambiente

Cumplen normas

Evitar accidentes laborables

Tecnología amigable

No genera efectos negativos al ambiente

Beneficios a la comunidad y al ambiente factible
No se generan desecho peligrosos

No se generan desecho peligrosos

Higiene, seguridad y ambiente

Empleo de técnicas o tecnologías sustentables

No se generan desecho peligrosos Factibilidad Beneficios

Fuente; Las Autoras, (2017) DP: Diseño de Planta; TMP: Transformación de Materias Primas; MOP: Mejoras Operacionales a Procesos; CE: Construcción de Equipos, ST: Sistemas de Tratamiento; pp: número de página en el proyecto

En el cuadro que a continuación observamos, el análisis realizado al contenido de los PSI del PNF de Química. 
Categorías emergentes del corpus texto de la unidad de análisis Viabilidad Ambiental (VA) en los PSI del PNF de $Q$.

\section{Cuadro 3}

$\begin{array}{ccc}\begin{array}{c}\text { Código de } \\ \text { PSI }\end{array} & \text { Unidad de Análisis } & \text { Categoría } \\ & & \text { Emergente }\end{array}$

\begin{tabular}{|c|c|c|c|}
\hline $\begin{array}{l}\text { 1-Q-2015- } \\
\text { TMP }\end{array}$ & $\begin{array}{l}\text { "este proyecto es factible ya que } \\
\text { desde la recolección de la materia } \\
\text { prima hasta el producto terminado no } \\
\text { se generan materiales o desechos } \\
\text { peligrosos o tóxicos al ambiente" }\end{array}$ & $\begin{array}{l}\text { Factible } \\
\text { No se generan desechos } \\
\text { peligrosos }\end{array}$ & $\begin{array}{l}\text { Factible } \\
\text { No se generan desecho } \\
\text { peligrosos }\end{array}$ \\
\hline $\begin{array}{l}\text { 2-Q-2015- } \\
\text { TMP }\end{array}$ & $\begin{array}{l}\text { "el proyecto es viable porque las } \\
\text { plantas que se usaran para el } \\
\text { suplemento alimenticio no han sido } \\
\text { tratadas con plaguicidas o con } \\
\text { fertilizantes" }\end{array}$ & $\begin{array}{l}\text { Es viable } \\
\text { Las plantas no han sido } \\
\text { tratada con plaguicidas y } \\
\text { fertilizantes }\end{array}$ & $\begin{array}{l}\text { Disponibilidad de } \\
\text { Materia prima natural }\end{array}$ \\
\hline $\begin{array}{l}\text { 3-Q-2015- } \\
\text { TMP }\end{array}$ & $\begin{array}{l}\text { "es viable porque se reduce la } \\
\text { contaminación ambiental, ya que se } \\
\text { aprovecha el lactoosuero para la } \\
\text { elaboración de otros productos que } \\
\text { antes era desechado directamente al } \\
\text { suelo o a los cuerpos de agua" }\end{array}$ & $\begin{array}{l}\text { Es viable } \\
\text { Reduce la contaminación } \\
\text { ambiental } \\
\text { Aprovechamiento de } \\
\text { desechos }\end{array}$ & $\begin{array}{l}\text { Resolución de un } \\
\text { problema } \\
\text { Empleo de técnicas o } \\
\text { tecnologías sustentables }\end{array}$ \\
\hline $\begin{array}{l}\text { 4-Q-2015- } \\
\text { OTROS }\end{array}$ & $\begin{array}{l}\text { "todo laboratorio genera } \\
\text { contaminación, así que de acuerdo a } \\
\text { los servicios que se presten en el } \\
\text { laboratorio se realizara un manual } \\
\text { para el manejo de desechos y } \\
\text { minimizar el impacto" }\end{array}$ & $\begin{array}{l}\text { Laboratorio } \\
\text { Generación de desechos } \\
\text { Manual para manejar los } \\
\text { desechos } \\
\text { Minimizar el impacto }\end{array}$ & $\begin{array}{l}\text { Medidas de control de } \\
\text { desechos generados }\end{array}$ \\
\hline $\begin{array}{l}\text { 5-Q-2015- } \\
\text { OTROS }\end{array}$ & $\begin{array}{l}\text { No aparece la unidad de análisis VA } \\
\text { en el PSI }\end{array}$ & & \\
\hline $\begin{array}{l}\text { 6-Q-2015- } \\
\text { TMP }\end{array}$ & $\begin{array}{l}\text { "puesto que el sustrato se hace con } \\
\text { estiércoles es una práctica de manejo } \\
\text { agronómica y económicamente } \\
\text { viable para la producción } \\
\text { sustentable" }\end{array}$ & $\begin{array}{l}\text { Agronómica y } \\
\text { económicamente viable } \\
\text { Producción sustentable }\end{array}$ & $\begin{array}{l}\text { Empleo de técnicas o } \\
\text { tecnologías sustentables }\end{array}$ \\
\hline $\begin{array}{l}\text { 7-Q-2015- } \\
\text { TMP }\end{array}$ & $\begin{array}{l}\text { "es positivo porque se emplean } \\
\text { técnicas agroecológica sustentable } \\
\text { para la comunidad" }\end{array}$ & $\begin{array}{l}\text { Es positivo } \\
\text { Técnicas agroecológica } \\
\text { sustentable }\end{array}$ & $\begin{array}{l}\text { Empleo de técnicas o } \\
\text { tecnologías sustentables }\end{array}$ \\
\hline $\begin{array}{l}\text { 8-Q-2015- } \\
\text { TMP }\end{array}$ & $\begin{array}{l}\text { "formular un abono tipo Bocashi } \\
\text { traerá múltiples beneficios a los }\end{array}$ & $\begin{array}{l}\text { Múltiples beneficios al } \\
\text { suelo }\end{array}$ & Beneficios \\
\hline
\end{tabular}


suelos y a la comunidad de DACA"

9-Q-2015-

TMP

"Estas bases puede sustituir a los

detergentes comerciales

representando una alternativa

ecológicamente viable porque son

biodegradables"

10-Q-2016- " "es viable porque se genera un

TMP

$11-\mathrm{Q}-2016-$ TMP

producto biodegradable a partir de un desecho"

"Se realizó una lista de chequeo para identificar los contaminantes en la zona de estudio, así como los reactivos necesarios para el proceso y los desechos generados en el laboratorio, evidenciándose un bajo impacto de las acciones emprendidas en la obtención biotecnológica del quitosano"..."se aplican medidas para mitigar el impacto"

12-Q-2016- "el proyecto es viable porque se TMP

13-Q-2017- “el proyecto es factible porque hay TMP formula un producto a base de plantas nativas que es inocuo al suelo" suficientes plantas silvestres en la zona de estudio"
Y a la comunidad

sustitución de detergentes comerciales

Resolución de un problema

alternativas ecológicamente viables biodegradables

Es viable

Aprovechamiento de Resolución de un problema

desechos

Productos biodegradables

Lista de chequeo

Aplicación de una

Identificación de contaminantes metodología para construir la VA

Fase de operación

Bajos impactos

Medidas de mitigación

Es viable

Producto inocuo al suelo

No produce daño al ambiente

Es factible

Suficientes plantas en la zona de estudio
Disponibilidad de Materia prima
Es factible

Fuente; Las Autoras, (2017) TMP: Transformación de Materias Primas; pp: número de página en el proyecto

Del Cuadro anterior se desprende que para el año 2015 los PSI del PNF-Q, enfoca la VA a la implementación de técnicas y/o tecnologías amigables con el ambiente, a la resolución de una problemática ambiental como el cambio a una situación esperada al aprovechar un desecho, el cual es transformado en un producto que traerá beneficios a la comunidad. Por otra parte, un grupo identifica corrientes de desechos y propone medidas para su control y mitigación. Por otro lado, un grupo asocia la VA con la disponibilidad de materia prima natural. En el año 2016, un solo grupo de proyecto aplica una metodología para construir la VA, la lista de chequeo, Identificando contaminantes solo en la fase de operación, concluyen que el PSI es viable porque los impactos son bajos y propone las medidas de mitigación, el resto de los proyectos realizados 
ese año relacionan VA con la resolución de un problema ambiental y con el hecho de no producir desechos que perjudiquen el ambiente. El PSI realizado en el año 2017, asocian la palabra factibilidad a la VA, y a la disposición de materias primas en la zona. Al contrastar esta información develada a través de los PSI, con las posibles metodología a aplicar según Arboleda (2008), pudimos advertir que en el PNF-PQ solo cinco PSI de los cuarenta y cinco leídos por su parte, en el PNF-Q un solo grupo de los 13 PSI, aplican alguna de las metodologías, entre las que se mencionan matriz de doble entrada, de Leopold, Delladova y la lista de chequeo. Al cotejar con los alcances del DAQ son estos mismos grupos los que cumplen con el alcance exigido, lo que nos sugiere que aplicar algunas de las metodologías asegura cumplir con los mencionados alcances.

\section{Lo emergente de la Interpretación}

Para iniciar este apartado conviene dejar claro que cualquier actividad humana interactúa de alguna manera con el entorno donde se emplazan, tanto en su construcción como en su operación. Es decir, generamos cambios en las condiciones ambientales, las cuales van a variar de acuerdo a la magnitud, la extensión o la duración del proyecto, es por eso que se debe analizar como tu proyecto va a influir en esas condiciones iniciales que encuentras en la comunidad, y como desde tu posición de gestor de proyecto vas a evitar esos efectos o en todo caso a mitigarlos o controlarlos, es aquí entonces donde radica la importancia de que la VA se realice de manera adecuada.

Como nos develo la investigación el grueso de los PSI (52 para ser exactos) no la construyen adecuadamente y tampoco cumplen con los alcances que se exigen en la guía orientadora. Un gran número de PSI entre los años 2012, 2013 y 2014 han relacionado la VA con los beneficios que traerá el proyecto, cuando hablamos de beneficios nos situamos en una situación postproyecto, por lo que nos da cuenta que no hay claridad conceptual de la VA, puesto que este es un análisis pre-proyecto. De igual manera, otros tienen la tendencia de relacionar la VA a la resolución de una problemática ambiental cuando probablemente su proyecto cause otro daño, pero como no lo han identificado a tiempo no se prevé, no se percatan del problema.

Es importante hacer hincapié que cuando vamos a la comunidad detectamos una problemática y proponemos una alternativa de solución, entonces es a esa solución, la que se propone, la que los participantes del proyecto deben desmenuzar para averiguar cómo podría afectar al medio donde 
emplazará tal propuesta. Por otro lado, hay grupos que consideran que si sus efluentes no son peligroso el proyecto es viable, hay que recordar toda actividad humana genera un efecto por lo que hay que evaluarlo a través del análisis de la VA. En esta misma tónica también hay quienes expresan que si resuelven el problema con una tecnología sustentable, ya pueden afirmar que el proyecto es viable, sin ni siquiera haber desglosado que la tecnología que proponen también genera un efecto. Por todo lo dicho hasta aquí, nos permite inferir que las fallas son recurrentes y las evidencias son los PSI revisados, los alcances que se exigen no se completan o simplemente no lo cumplen, por descuido, desconocimiento o por flexibilidad, se está construyendo inadecuadamente la VA.

Para comprender el significado que los estudiantes le otorgaron a la VA, partimos en primer lugar del deber ser para los participantes del PNF-PQ deben caracterizar de forma física-natural la zona de estudio e identificar contaminantes en la zona, esto concuerda con la naturaleza de sus proyecto puesto que la mayoría se destinan al DP y a la $\mathrm{CE}$, pero al parecer este paso no lo cumplen porque probablemente no se tiene clara la visión del área que van a intervenir ni el lugar de la comunidad donde ubicarían la planta o el equipo, para el segundo alcance: Identificación de efluentes contaminantes y sustancias toxicas, algunos lo hace específicamente los que CE o hacen las MOP, pero indican que al no ser peligroso no perjudicará al ambiente, en esta parte hay que dejar claro que todo efluente sea peligroso o no, sea líquido, gaseoso o sólido genera un impacto por lo que hay que tomarlo en cuenta, además de ello consideramos importante añadir a la guía orientadora los afluentes, es decir las corrientes de entrada a la planta o al equipo, también debe considerase su análisis $\mathrm{p}$ a la guía orientadora los afluentes puesto que éstos pueden ser recursos naturales o materia primas que causen alguna degradación o contaminación.

Para el tercer alcance: Prevé las medidas de control y saneamiento ambiental, estos casi nadie los cumple, y es coherente porque si no han identificado contaminantes que medidas han de contemplar para el control. Por último: Presenta alternativas de procesos para los posibles tratamientos de efluentes y sólidos contaminantes, aquí aplica el mismo comentario realizado en el alcance anterior. Con respecto al PNF-Q sus PSI se orientan en su gran mayoría a la TMP, para formular productos de interés comercial, ahora bien su VA, se direcciona en su gran mayoría al trabajo que realizan dentro del laboratorio en la síntesis de esos productos, hay consumo de agua, de energía eléctrica, de reactivos químicos, de materias primas y por lo tanto se generan aguas de lavado, aguas residuales, subproductos de síntesis, materiales descartables de laboratorio entre 
otros, que ninguno de los PSI tenían presente en la VA. Hay dos PSI que de forma incipiente Identificación de los posibles contaminantes en la comunidad y/o de la materia prima para cumplir con el primer alcance, pero ¿porque no concluyeron con el resto de los alcances? (Especificar los contaminantes de la materia prima y los posibles a generar en el producto terminado (subproductos-derivados de la síntesis).

Tomar en cuenta en el desarrollo del proyecto las medidas de control y saneamiento ambiental, Presenta alternativas para el tratamiento de los desechos generados en la ejecución del nuevo producto y/o método desarrollado) esto nos da un indicio de cierta incongruencia entre lo que está escrito en la guía orientadora y lo que verdaderamente se hace en los PSI, resultando estos una evidencia de un conjunto de conocimientos que no han sido debidamente organizados, así como pertinentemente explicados, develando un patrón de coincidencias entre los PSI revisados y puntos de desencuentros de éstos con los referentes teóricos y con la guía orientadora de proyecto del DAQ.

El ambiente es un eje transversal en el currículo de la UPTAG, con la realidad descubierta en esta investigación a través de los PSI que se han venido realizando en el DAQ, bajo los dos PNF que se administran Q y PQ, cabe Preguntarse: ¿Realmente la transversalidad se logra? ¿Cuál será la realidad en los otros PNF? En este sentido entra en juego, una respuesta que se vincula directamente a la formación de los docentes en materia ambiental, un tema cacareado de vieja data sin embargo el mismo no ha sido resuelta, hay que sumar voluntades para formarnos en colectivo y verdaderamente cumplir el rol de docente mediador del conocimiento pertinente con acción social y ambiental.

Igualmente cabe reflexionar sobre la postura que asumen los participantes de los PSI, al construir la VA, debe tener el significado de aportar elementos e información importante sobre que recursos que encuentran en las comunidades, los cuales deben identificarse, reconocerse, convirtiéndose la VA para los autores en un enlace entre las actividades del proyecto capaces de degradar esos recursos naturales y la posibilidad de mitigar o eliminar esos efectos, de tal manera que los autores están previniendo el deterioro de los recursos naturales antes de que ocurran, lo que se trasciende positivamente en el ambiente, en la relación Proyecto-ambiente en las Comunidades y en la formación de la conciencia ecologista y responsable de estos profesionales egresados de la UPTAG. Para finalizar hay que hacer mejoras en la guía orientadora para que 
pueda cumplir su propósito y recordar que los cambios que se generan a partir de una evaluación y una autorreflexión crítica de los aciertos y desaciertos nos permiten crecer como profesionales.

\section{Referencias consultadas}

1. Arboleda, J. (2008). Manual de evaluación ambiental de actividades, obras y proyectos. Medellín Colombia.

2. Díaz, C y Navarro, P. (1998). “Análisis de contenido", en: Métodos y técnicas cualitativas de investigación en ciencias sociales. Segunda reimpresión. Madrid, Editorial Síntesis, SA. Departamento Académico de Química. Guía Orientadora para la elaboración de los PSI.

3. Gadamer, H. (1996). Verdad y método I. Madrid: Sígueme

4. Guanipa Gotopo, C. (2018). Aproximación Teórica-epistemológica de la Gestión Curricular, en el contexto de las Universidades Politécnicas Territoriales. Revista Arbitrada Interdisciplinaria Koinonía, 3(5), 27-52. Recuperado de http://fundacionkoinonia.com.ve/ojs/index.php/revistakoinonia/article/view/112/94

5. Ley Orgánica de Educación (2009) [Transcripción en línea]. [Consulta: 2017, mayo 28]. Disponible en: http://www.me.gob.ve/ley_organica.pdf

6. Ministerio del Poder Popular para la Educación Universitaria. (2012) Lineamientos para el Desarrollo Curricular de los Programas Nacionales de Formación. Caracas, Venezuela.

7. Ocampo E. (2003); Costos y Evaluación de Proyectos; CECSA UNAM, 2da Edición.

8. Ortegón, E. (2005). Metodología General de Preparación, Evaluación de Proyectos de Inversión Pública; CEPAL.

9. Real Academia Española. 2001. Diccionario de la lengua española (Edición 22 a . ed. Madrid, España: Autor. 
10. Silva, J. (2007). Metodología de la investigación: elementos básicos. Caracas: Co-Bo

11. Sobrero, F. (2009). Análisis de Viabilidad: La cenicienta en los Proyectos de Inversión. FCE -UNL

12. Tójar, J. (2006). Investigación cualitativa comprender y actuar. Madrid: La Muralla S.A

13. Varsavsky, O. (2007) Ciencia, política y cientificismo. Ediciones Ministerio del Poder Popular para Ciencia y tecnología. Monte Ávila Editores Latinoamericana. Caracas, Venezuela.

\section{References consulted}

1. Arboleda, J. (2008). Manual of environmental evaluation of activities, works and projects. Medellin Colombia.

2. Díaz, C and Navarro, P. (1998). "Content analysis", in: Qualitative methods and techniques of research in social sciences. Second reprint Madrid, Editorial Síntesis, SA. Academic Department of Chemistry. Guidance Guide for the development of PSI.

3. Gadamer, H. (1996). Truth and method I. Madrid: Follow me

4. Guanipa Gotopo, C. (2018). Theoretical-epistemological approach to Curriculum Management, in the context of Territorial Polytechnic Universities. Interdisciplinary Arbitrated Review Koinonía, 3 (5), 27-52. Recovered from http://fundacionkoinonia.com.ve/ojs/index.php/revistakoinonia/article/view/112/94

5. Organic Law of Education (2009) [Online transcript]. [Consultation: 2017, May 28]. Available at: http://www.me.gob.ve/ley_organica.pdf

6. Ministry of Popular Power for University Education. (2012) Guidelines for the Curricular Development of National Training Programs. Caracas Venezuela. 
7. Ocampo E. (2003); Costs and Project Evaluation; CECSA UNAM, 2nd Edition.

8. Ortegón, E. (2005). General Methodology of Preparation, Evaluation of Public Investment Projects; ECLAC

9. Royal Spanish Academy. 2001. Dictionary of the Spanish language (Edition 22.a. ed. Madrid, Spain: Author.

10. Silva, J. (2007). Research methodology: basic elements. Caracas: Co-Bo

11. Sobrero, F. (2009). Feasibility Analysis: Cinderella in Investment Projects. FCE -UNL

12. Tójar, J. (2006). Qualitative research understand and act. Madrid: La Muralla S.A

13. Varsavsky, O. (2007) Science, politics and scientism. Editions Ministry of Popular Power for Science and Technology. Monte Ávila Latin American Publishers. Caracas Venezuela.

\section{Referências consultadas}

1. Arboleda, J. (2008). Manual de avaliação ambiental de atividades, obras e projetos. Medellín - Colômbia.

2. Díaz, C e Navarro, P. (1998). "Análise de conteúdo", em: Métodos e técnicas qualitativas de pesquisa em ciências sociais. Segunda reimpressão Madrid, Editorial Síntesis, SA. Departamento Acadêmico de Química. Guia de orientação para o desenvolvimento de PSI.

3. Gadamer, H. (1996). Verdade e método I. Madrid: Siga-me

4. Guanipa Gotopo, C. (2018). Abordagem teórico-epistemológica da Gestão Curricular, no contexto das Universidades Politécnicas Territoriais. Revisão Interdisciplinar Arbitrada 
Koinonía, $\quad 3 \quad$ (5), $\quad$ 27-52. $\quad$ Recuperado de http://fundacionkoinonia.com.ve/ojs/index.php/revistakoinonia/article/view/112/94

5. Lei Orgânica da Educação (2009) [Transcrição Online]. [Consulta: 2017, 28 de maio]. Disponível em: http://www.me.gob.ve/ley_organica.pdf

6. Ministério do Poder Popular para a Educação Universitária. (2012) Diretrizes para o desenvolvimento curricular de programas nacionais de treinamento. Caracas Venezuela.

7. Ocampo E. (2003); Avaliação de Custos e Projetos; CECSA UNAM, 2 $2^{\mathrm{a}}$ Edição.

8. Ortegón, E. (2005). Metodologia Geral de Preparação, Avaliação de Projetos de Investimento Público; CEPAL

9. Real academia espanhola. 2001. Dicionário da língua espanhola (Edição 22.a. ed. Madri, Espanha: Autor.

10. Silva, J. (2007). Metodologia de pesquisa: elementos básicos. Caracas: Co-Bo

11. Sobrero, F. (2009). Análise de Viabilidade: Cinderela em Projetos de Investimento. FCE $-\mathrm{UNL}$

12. Tójar, J. (2006). Pesquisa qualitativa entender e agir. Madri: La Muralla S.A

13. Varsavsky, O. (2007) Ciência, política e cientificismo. Edições Ministério do Poder Popular de Ciência e Tecnologia. Editores da América Latina Monte Ávila. Caracas Venezuela.

(C2019 por los autores. Este artículo es de acceso abierto y distribuido según los términos y condiciones de la licencia Creative Commons Atribución-NoComercial-CompartirIgual 4.0 Internacional (CC BY-NC-SA 4.0) (https://creativecommons.org/licenses/by-nc-sa/4.0/). 